\title{
NEISSERIA GONORRHOEAE IN A PREPUBERTAL CHILD
}

\author{
Lisa J. PFITZER
}

University of Louisville, Division of Pediatric Forensic Medicine, Louisville, Kentucky, USA e-mail: lisa.pfitzer@louisville.edu; tel.: + 502-629-3099; fax.: + 502-629-3096
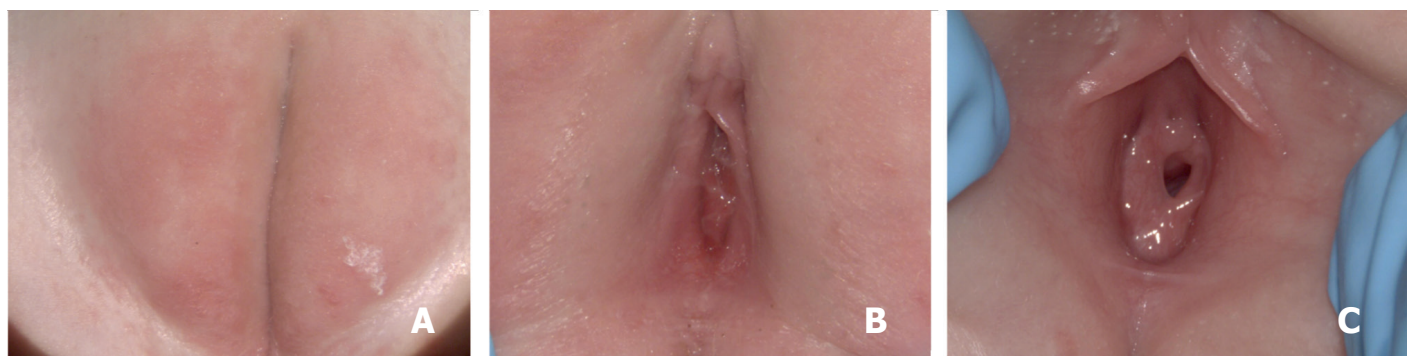

This two and a half year old female had been in the care of an adult male who was being investigated for sexual abuse based on the disclosure of an older child. On the morning of the child's appointment the mother noticed a green discharge on the child's panties. On examination, the labia majora were markedly erythematous (Panel A). Green discharge was present on the panties. A white mucoid discharge at the vaginal area was noted. The mucosa appeared edematous. The hymenal rim was not well demarcated (Panel B), presumably due to swelling. The rest of the exam was normal. A culture of the vaginal discharge was positive for gonorrhea. Gonorrhea in a toddler is highly suggestive of sexual abuse and confirms mucosal contact with infective secretions. The child received a ceftriaxone injection. A follow up examination revealed genital mucosa without discharge or edema. The hymenal rim was normal without tears or scars (Panel C). It is possible that infection is transmitted with exchange of body fluids by intimate touching, but not necessarily intercourse, where child victims have described the offender rubbing their genitals on genital areas. Most child sexual abuse victims will have a normal exam. Follow up labs were negative. The offender's testing status was unknown. The case was reported to child protection and law enforcement.

Key words: Gonorrhea - Child sexual abuse

Received: July 20, 2012

Accepted: September 18, 2012

Copyright (C) 2013 by University Clinical Center Tuzla.

E-mail for permission to publish: paediatricstoday@ukctuzla.ba

Citation: Pfitzer JL. Neisseria gonorrhoeae in a prepubertal child. Paediatrics Today. 2013;9(1):54. 